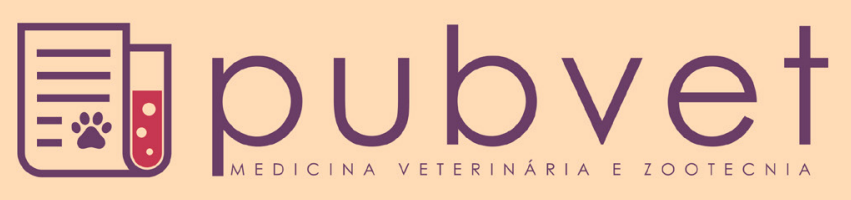

HTTP://DX.DOI.ORG/10.22256/PUBVET.V11N4.327-332

\title{
Análise da utilização da pimenta (Capsicum frutescens L.) e sua indicação medicinal: Revisão
}

\author{
Carolina Moreira de Santana ${ }^{1}$, Francileuda Batista de Almeida ${ }^{1}$, Francisco Regis da \\ Silva $^{1^{*}}$, Wyara Ferreira Melo ${ }^{1}$, Geraldo Gonzalez Talabera ${ }^{2}$, Weslley Epifanio Sarmento ${ }^{1}$, \\ Wenya Sarmento Sobrinho ${ }^{3}$, Paulo César Ferreira Linhares ${ }^{4}$
}

\footnotetext{
${ }^{I}$ Mestranda em Sistemas Agroindustriais, Universidade Federal de Campina Grande (UFCG), Pombal, PB, Brasil. E-mail: santanacarolina@hotmail.com; farmaciasantamariacz@gmail.com; wyarafmelo@gmail.com; weslleyep@yahoo.com.br

${ }_{2}^{2}$ Médico, Instituto de Ciências Médicas de Havana; Especialista em Saúde da Familia pela Universidade Federal do Ceará (UFC), Morada Nova, CE, Brasil..E-mail: geraldogonzaleztalabera@yahoo.es;

${ }^{3}$ Graduando em Serviço Social, Faculdade de Filosofia, Ciências e Letras de Cajazeiras (FAFIC), Cajazeiras, PB, Brasil. E-mail: wenvasarmento@hotmail.com;

${ }^{4}$ Doutor em Fitotecnia pela Universidade Federal Rural do Semi-Arido (UFERSA), Mossoró, RN, Brasil; Professor colaborador do Programa de Pós-Graduação em Sistemas Agroindustriais (PPGSA/CCTA), Universidade Federal de Campina Grande (UFCG), Pombal, PB, Brasil; E-mail:paulolinhares@ufersa.edu.br.

*Autor para correspondência: regisfrs@hotmail.com
}

RESUMO. A utilização de hortaliças no tratamento de doenças e na recuperação da saúde é um método terapêutico natural que está presente de forma marcante na cultura popular. Assim, o uso de remédios caseiros é uma das providências imediatas mais adotadas no tratamento de várias doenças. Diante disso, o uso de folhas e do fruto de planta Capsicum spp. (pimenta), foi analisado nesse artigo a fim de verificar o seu potencial terapêutico, através de uma revisão de literatura. A pesquisa se trata de uma revisão bibliográfica, abordando a importância da pimenta e a suas propriedades farmacológicas, utilizando-se de artigos e outras publicações. Para a realização desse trabalho buscou-se artigos originais e revisões indexadas nas bases SciELO, Periódicos CAPES, PubMed e MEDLINE, que abordava os temas: pimenta, pimenta e propriedade funcional, pimenta e atividade farmacológica. Não houve restrição quanto ao ano de publicação. A utilização do princípio ativo à base de plantas medicinais pode ser considerada como recursos auxiliares em um programa terapêutico global, podendo seu uso ser buscado cada dia mais na utilização de terapia paliativa dos cuidados da saúde. Assim, a literatura pesquisada elucidou que a pimenta tem grande valor do ponto de vista medicinal, além de representar importância cultural e econômica.

Palavras chave: Capsicum, pimenta, terapêutica, saúde

\section{Analysis of the use of pepper (Capsicum frutescens L.) and its medical indication: Review}

ABSTRACT. The use of vegetable crops in the treatment of diseases and the health
recovery is a therapeutic method that is present markedly in popular culture. Thus, the use
of homemade remedies is one of the immediate action more adopted in the treatment of
various diseases. In addition, the use of leaves and fruit of plant Capsicum spp. (pepper),
was analyzed in this article in order to verify your therapeutic potential, through a literature
review. The research is a literature review, focusing on the importance of pepper and its
pharmacological properties, using articles and other publications. For the realization of this
work sought to original articles and reviews are indexed in the SciELO, CAPES
Periodicals, PubMed and MEDLINE, which addressed the following themes: pepper,
pepper and functional property, pepper and pharmacological activity. There was no 
restriction regarding the year of publication. The use of the active principle based on medicinal plants can be considered an auxiliary resource in a therapeutic program global, which its use be sought more and more each day in the use of palliative therapy of health care. Thus, the literature explained that the pepper has great value from the point of view of medicinal, in addition to representing cultural importance and cost.

Key words: Capsicum, Chili, Therapeutic, Health

\section{Análisis de la utilización de pimienta (Capsicum frutescens L.) y su indicación médica: Revisión}

RESUMEN. El uso de hortalizas en el tratamiento de enfermedades y en la recuperación de la salud es un método terapéutico natural que está presente marcadamente en la cultura popular. Por lo tanto, el uso de remedios caseros es una de las acciones inmediatas más adoptadas en el tratamiento de diversas enfermedades. Frente a eso, el uso de hojas y de frutos de la planta Capsicum spp. (Pimienta), se analizó en este artículo, con el fin de verificar su potencial terapéutico. La investigación se trata de una revisión de literatura, abordando la importancia de la pimienta y sus propiedades farmacológicas. Para la realización de esta actividad se consultaron artículos originales y revisiones indexadas en las bases de datos SciELO, Revistas CAPES, PubMed y MEDLINE, que abordaron los siguientes temas: pimienta, pimienta y propiedad funcional, la pimienta y actividad farmacológica. No hubo restricción referente al año de publicación. El uso del principio activo a base de plantas medicinales se puede considerar como recursos auxiliares en un programa terapéutico integral, pudiendo su utilización ser buscado cada día más en el uso de terapia paliativa del cuidado de la salud. De este modo, la literatura ha dilucidado que la pimienta tiene un gran valor desde el punto de vista medicinal, además de representar la importancia cultural y económica.

Palabras clave: Capsicum, pimienta, terapia, salud

\section{Introdução}

A utilização de hortaliças no tratamento de doenças e na recuperação da saúde é um método terapêutico natural que está presente de forma marcante na cultura popular (Lorenzi et al., 2002), fato que tem levado órgãos oficiais de saúde pública no Brasil à implantação de Políticas que viabilizem a utilização racional, segura e eficaz de plantas medicinal e fitoterápico (BRASIL, 2006a; 2006b).

A Organização Mundial de Saúde refere-se às plantas medicinais ou parte da planta como "espécies vegetais a partir das quais produtos de interesse terapêutico podem ser obtidos e usados na espécie humana como medicamento" (Di Stasi, 2007). Portanto, são plantas, parte dela ou frutos que produzem substâncias químicas farmacológicas ativas para o organismo humano e quando administradas exercem função terapêutica.

No entanto, todas as espécies vegetais possuem componentes químicos, muitos dos quais podem ser ativos como medicamento, mas isto não torna a espécie uma planta medicinal. Na verdade, muitos autores defendem que as plantas medicinais são aquelas reconhecidas pela população como uma espécie que tem valor medicinal, ou seja, que tem alguma propriedade que serve para prevenir ou combater determinadas doenças (Nolla et al., 2005, Di Stasi, 2007). A planta é considerada pela população como medicinal caso ela seja eficaz na prevenção ou tratamento de uma doença ou para alívio de um sintoma. O que não é possível saber é se o uso da planta é suficiente para tratar a doença ou malestar desejado ou se a planta usada como remédio será melhor ou mais potente que um outro medicamento da farmácia e se será segura o suficiente para o uso geral (Di Stasi, 2007).

Comercialmente o uso de plantas medicinais é bastante conhecidos e discutidos no Brasil e no mundo. O mercado atende de diferentes formas o consumidor desse tipo de produto, incluindo as comercializações feitas em empresas, em mercados e em ervanários (Brandão et al., 2003).

Assim, o uso de remédios caseiros é uma das providências imediatas mais adotadas no tratamento de várias doenças. Diante disso, o uso de folhas e do fruto de planta Capsicum spp. 
(pimenta), foi analisado nesse artigo a fim de verificar o seu potencial terapêutico, através de uma revisão de literatura.

\section{Metodologia}

A pesquisa se trata de uma revisão bibliográfica, abordando a importância da pimenta e a suas propriedades farmacológicas, utilizandose de artigos e outras publicações.

Para a realização desse trabalho buscou-se artigos originais e revisões indexadas nas bases SciELO, Periódicos CAPES, PubMed e MEDLINE, que abordava os temas: pimenta, pimenta e propriedade funcional, pimenta e atividade farmacológica. Não houve restrição quanto ao ano de publicação.

\section{Resultados e Discussão}

\section{Importância da pimenta}

Capsicum L. é o gênero botânico da família Solanaceae, que abrange as pimentas e os pimentões, hortícolas originários da América Central e do Sul. Acredita-se que a exploração de tais pimentas se deu desde o início do povoamento humano nas Américas, supostamente há cerca de 12.000 anos (Bosland et al., 2000). Por conta desse largo curso de tempo, populações ameríndias descobriram modos de satisfazer, por meio desses vegetais, necessidades diversas, como as ligadas à alimentação e à cura de doenças. Incluíram-nos em cultos sagrados e nos arredores de suas habitações como plantas ornamentais (Bosland et al., 2000, Nuez et al., 1996, Bosland, 1999).

Segundo Landrum (1986), as espécies do gênero Pimenta apresentam odores característicos, principalmente nas folhas, devido à presença de óleos essenciais que conferem sabor picante e odor forte.

O gênero Pimenta tem sido bastante estudado devido às suas propriedades biológicas, que incluem atividade antimicrobiana, antiinflamatória, analgésica, antipirética, antinociceptiva e hipotensiva, entre outras (Custódio et al., 2010, Paula et al., 2010).

No estudo de Furtado et al. (1978), entre pescadores do litoral paraense, encontra-se citado o emprego da planta para o amadurecimento de tumores. Em Roraima registras-se seu uso no tratamento de 'pano-branco', conforme notas de Van den Berg and Silva (1988). Quilombolas do Amapá conhecem modos de aproveitar as pimentas para aliviar cólicas menstruais e de crianças e para os casos de dores reumáticas e problemas intestinais, conforme Pereira et al. (2007). Entre índios Yanomami, os estudos de Milliken and Albert (1997) e de Milliken et al. (1999) registram o emprego da pimenta para tratar infecções respiratórias, oftalmias e malária. Índios colombianos as empregavam no tratamento de picadas de cobras (Otero et al., 2000). Povos maias e astecas usavam-nas, misturadas com milho, para cura de resfriados comuns. Também as utilizavam em casos de queimaduras e no tratamento de asmas, tosses e dores de garganta (Cichewicz and Thorpe, 1996, Bosland et al., 2000).

No estudo de Roman et al. (2011), os autores fizeram um levantamento sobre a utilização medicinal da pimenta e das partes da planta com os moradores de uma comunidade no município de Santarém- PA, como segue o resultado no quadro abaixo (Quadro 1). Neste, encontra-se as principais indicações medicinais da pimenta malagueta $C$. frutescens na comunidade de Cabeça D’Onça, município de Santarém, Pará.

Quadro 1. Indicações medicinais da pimenta malagueta $C$. frutescens na comunidade de Cabeça D'Onça, Santarém, Pará.

\begin{tabular}{|l|c|}
\hline Indicações & Partes usadas \\
\hline Pano-branco, titinga, mancha no corpo & Folha \\
\hline Dor de dente & Fruto \\
\hline Reumatismo & Fruto \\
\hline Erisipela, vermelha, vermelhão & Folha \\
\hline Impinge & Folha \\
\hline Furúnculos & Folha \\
\hline Amebas e vermes & Fruto \\
\hline Coceira & Folha \\
\hline Inchaço & Folha \\
\hline Ferrada de arraia & Fruto \\
\hline Coração & Fruto \\
\hline Derrame & Folha e fruto \\
\hline Hemorroida & Fruto \\
\hline Problemas de parto & Fruto \\
\hline Queda de cabelo & Folha \\
\hline
\end{tabular}

Fonte: Roman et al. (2011).

\section{Propriedades farmacológicas}

Uma planta medicinal ou o respectivo extrato, como qualquer outro medicamento, deve passar por um processo de validação, que inclui a comprovação da atividade farmacológica e da possível toxicidade em humanos (Cervo et al., 2007).

Até por volta do século XIX as plantas medicinais e derivados consistiam na base da 
terapêutica. Com o advento da pesquisa científica, muitas substâncias puderam ser isoladas das plantas e serviram de modelos para a produção de fármacos (Yunes et al., 2007). As matérias prima vegetais são utilizadas na produção de preparações caseiras para tratar as mais diversas doenças, e ainda como matéria-prima para a indústria farmacêutica, o que representa proporção substancial do mercado global de medicamentos (WHO, 1998). Estima-se que $80 \%$ da população mundial fazem uso das plantas como primeiro recurso terapêutico (Silva Junior et al., 2010).

A propriedade analgésica dos capsaicinóides, produzidas pela placenta do fruto de Capsicum, tem sido também explorada pela indústria farmacêutica. A capsaicina, o alcalóide mais estudado destas solanáceas, vem sendo empregada na elaboração de analgésicos tópicos para o tratamento de artrites, herpes zoster, diabetes neuropáticas, neuralgias, dores pós-cirúrgicas, como anti-inflamatório, agente anticancerígeno, entre outros. O uso externo dessa substância, por meio de creme e pomadas, diminui a dor, pois atua na inibição do acúmulo, dentro do neurônio, de um neurotransmissor chamado 'substância P', responsável pela sensação de dor. Ou seja, ela impede que os nervos emitam sinais de dor ao cérebro. Além disso, a sensação de calor que os capsaicinóides provocam no organismo auxilia no tratamento de dores musculares e das articulações (Eshbaugh, 1993, Sampaio and Rivitti, 2000, Berke and Shieh, 2001, Carvalho et al., 2001).

Há na literatura referência ao emprego das folhas de Capsicum como antibiótico, carminativo, rubefaciente, estimulante, estomáquico e vesicante (Molina-Torres et al., $\underline{1999}$, Bosland et al., 2000, Barros and Napoleão, 1998).

A P. pseudocaryophyllus, uma espécie da Capsicum, apresenta composição química complexa, tendo como característica a presença de compostos fenólicos, taninos e flavonoides, o que sugere importante potencial fitoterapêutico a ser investigado, além de apresentar traços de heterosídeos antraquinônicos (Paula et al., 2008, El Assal, 2012). A atividade antimicrobiana e antifúngica do extrato obtido de folhas de $P$. pseudocaryophyllus tem sido amplamente testada.

$\mathrm{Na}$ folha da Capsicum se encontra a eugenol, que possuí uma atividade antisséptica, registramse diversas finalidades do eugenol na indústria, incluindo flavorizante em produtos alimentícios, especialmente em carnes e salsichas, sendo também usado em condimentos. É também usado na fabricação de perfumes, cosméticos e sabonetes, também servindo de matéria prima para a produção do isoeugenol, que por sua vez é usado na fabricação da vanilina (baunilha).

\section{Considerações Finais}

A utilização do princípio ativo à base de plantas medicinais pode ser considerada como recursos auxiliares em um programa terapêutico global, podendo seu uso ser buscado cada dia mais na utilização de terapia paliativa dos cuidados da saúde.

A sistematização do conhecimento a respeito da espécie pretende valorizar e preservar os conhecimentos tradicionais que, aliados ao conhecimento científico, possam promover o uso racional da espécie como recurso econômico e terapêutico.

Assim, a pimenta tem grande valor do ponto de vista medicinal, além de representar importância cultural e econômica. Uma maior investigação a respeito das propriedades medicinais da espécie que inclua estudos de sua toxicidade e segurança é necessária, uma vez que a utilização racional de plantas com fins terapêuticos é de grande valor no setor de saúde em países em desenvolvimento.

\section{Referências Bibliográficas}

Barros, J. F. P. \& Napoleão, E. 1998. Ewé Òrìsà: Uso litúrgico e terapêutico dos vegetais nas casas de candomblé Jêje-Nago. Betrand Brasil. Rio de janeiro.

Berke, T. G. \& Shieh, S. C. 2001. Capsicum, Chillies, Paprika, Bird's Eye Chilli. In: Peter, K. V. (ed.) Handbook of Herbs and spices. Cambridge: Woodhead, New York.

Bosland, P. W. 1999. Chiles: a gift from a fiery God. Hort Science, 34, 809-811.

Bosland, P. W., Votava, E. J. \& Votava, E. M. 2000. Peppers: vegetable and spice capsicums. Cabi.

Brandão, M. G. L., Freire, N. \& Vianna-soares, C. D. 2003. Vigilância de fitoterápicos. In: Cabrera, L. \& Chavagnac, B. (eds.) La forêt et lês dieux: religions afro-cubaines et médecine sacrée à Cuba. J.-M. Place, Paris.

BRASIL. Decreto n. 5.813, de 22 de junho de 2006. Aprova a Política Nacional de Plantas Medicinais e Fitoterápicos e dá outras 
providências. Diário Oficial da União, Brasília, DF, 23 de junho de 2006a.

BRASIL. Ministério da Saúde. Portaria n. 971, de 3 de maio de 2006. Aprova a Política Nacional de Práticas Integrativas e Complementares (PNPIC) no sistema Único de saúde. Diário Oficial da União, Brasília, DF, 4 de maio de 2006b.

Carvalho, J. C. T., Gosmann, G., Schenkel, E. P. \& Simões, C. M. O. 2001. Compostos fenólicos simples e heterosídicos. In: Simões, C. M. O., Schinkel, E. P., Gosmann, G., Mello, J. C. P., Mentz, L. A. \& Ros Petrovick, P. (eds.) Farmacognosia: da planta ao medicamento. Editora UFRGS, Florianópolis.

Cervo, A. L., Bervian, P. A. \& Silva, R. 2007. Metodologia científica, 6 ed. edn. Pearson Prendice Hall, São Paulo.

Cichewicz, R. H. \& Thorpe, P. A. 1996. The antimicrobial properties of chile peppers (Capsicum species) and their uses in Mayan medicine. Journal of Ethnopharmacology, 52, 61-70.

Custódio, D. L., Burgo, R. P., Moriel, B., Barbosa, A. M., Rezende, M. I., Daniel, J. F. d. S., Pinto, J. P., Bianchini, E. \& Faria, T. J. 2010. Antimicrobial activity of essential oils from Pimenta pseudocaryophyllus and Tynanthus micranthus. Brazilian Archives of Biology and Technology, 53, 1363-1369.

Di Stasi, L. C. 2007. Plantas medicinais verdades e mentiras: o que os usuários e os profissionais de saúde precisam saber. UNESP, São Paulo.

El Assal, F. E. 2012. Bioatividade in vitro de Pimenta pseudocaryophyllus sobre Candida spp e complexo. Planta, 256, 128.

Eshbaugh, W. H. 1993. Peppers: History and exploitation of a serendipitous new crop discovery. In: Janick, J. \& Simon, J. E. (eds.) New Crops. John Wiley \& Sons, New York.

Furtado, L. G., Berg, M. E. \& Souza, R. C. 1978. Notas sobre o uso terapêutico de plantas pela população cabocla de Marapanim, Pará. Boletim Museu Paraense Emílio Goeldi, Nova Série Antropologia, 70, 1-31.

Landrum, L. R. 1986. Campomanesia, Pimenta, Blepharocalyx, Legrandia, Acca, Myrrhinium, and Luma (Myrtaceae). Flora Neotropica, 45, $1-178$
Lorenzi, H., Matos, F. J. \& Francisco, J. M. 2002. Plantas medicinais no Brasil: nativas e exóticas.

Milliken, W. \& Albert, B. 1997. The use of medicinal plants by the Yanomami Indians of Brazil, Part II. Economic Botany, 51, 264-278.

Milliken, W., Albert, B., Gomez, G. G. \& Rutherford, J. 1999. Yanomami: A forest people. Royal Botanic Gardens, Kew.

Molina-Torres, J., García-Chávez, A. \& RamírezChávez, E. 1999. Antimicrobial properties of alkamides present in flavouring plants traditionally used in Mesoamerica: affinin and capsaicin. Journal of Ethnopharmacology, 64, 241-248.

Nolla, D., Severo, B. M. A. \& Micott, A. M. B. M. 2005. Plantas medicinais. Universidade Federal de Pelotas, Passo Fundo.

Nuez, V., Ortega, F. \& Joaquín, R. C. G. 1996. El cultivo de pimientos, chiles y ajíes. MundiPrensa, Madrid.

Otero, R., Núñez, V., Barona, J., Fonnegra, R., Jiménez, S. L., Osorio, R. G., Saldarriaga, M. \& Diaz, A. 2000. Snakebites and ethnobotany in the northwest region of Colombia: Part III: Neutralization of the haemorrhagic effect of Bothrops atrox venom. Journal of Ethnopharmacology, 73, 233-241.

Paula, J. A. M., Paula, J. R., Bara, M. T. F., Rezende, M. H. \& Ferreira, H. D. 2008. Estudo farmacognóstico das folhas de Pimenta pseudocaryophyllus (Gomes) LR LandrumMyrtaceae. Revista Brasileira de Farmacognosia, 18, 265-278.

Paula, J. A. M., Reis, J. B., Ferreira, L. H. M., Menezes, A. C. S. \& Paula, J. R. 2010. Gênero Pimenta: aspectos botânicos, composição química e potencial farmacológico. Revista Brasileira de Plantas Medicinais, 12, 363-379.

Pereira, L. A., Lima, R. B., Guimarães, E. F., Almeida, M. Z., Monteiro, E. D. C. Q. \& Sobrinho, F. d. A. P. 2007. Plantas medicinais de uma comunidade quilombola na Amazônia Oriental: Aspectos utilitários de espécies das famílias Piperaceae e Solanaceae. Cadernos de Agroecologia, 2, 1385-1388.

Roman, A. L. C., Ming, L. C., Carvalho, I. \& Sablayrolles, M. d. G. P. 2011. Uso medicinal da pimenta malagueta (Capsicum frutescens L.) em uma comunidade de várzea à margem 
do rio Amazonas, Santarém, Pará, Brasil. Boletim do Museu Paraense Emilio Goeldi. Ciências Humanas, 6, 543-557.

Sampaio, S. A. P. \& Rivitti, E. A. 2000. Dermatologia. Artes Médicas, São Paulo.

Silva Junior, I. F., Baker, D., Raimondi, M., Zacchino, S., Cechinel Filho, V., Noldin, V. F., Rao, V. S., Lima, J. \& Martins, D. T. 2010. Evaluation of the antifungal activity and mode of action of Lafoensia pacari A. St.-Hil., Lythraceae, stem-bark extracts, fractions and ellagic acid. Revista Brasileira de Farmacognosia, 20, 422-428.

Van den Berg, M. E. \& Silva, M. H. L. 1988. Contribuição ao conhecimento da flora medicinal de Roraima. Acta amazônica, 18, 23-35.

WHO. 1998. Quality control methods for medicinal plant materials. World Health Organization, Geneve.
Yunes, R. A., Cechinel Filho, V., Yunes, R. A. \& Cechinel Filho, V. 2007. Novas perspectivas dos produtos naturais na química medicinal moderna. In: Yunes, R. A. (ed.) Química de produtos naturais, novos fármacos ea moderna farmacognosia. UNIVALI, Itajaí.

\section{Article History:}

Received 8 January 2017

Accepted 17 January 2017

Available on line 10 February 2017

License information: This is an open-access article distributed under the terms of the Creative Commons Attribution License 4.0, which permits unrestricted use, distribution, and reproduction in any medium, provided the original work is properly cited 